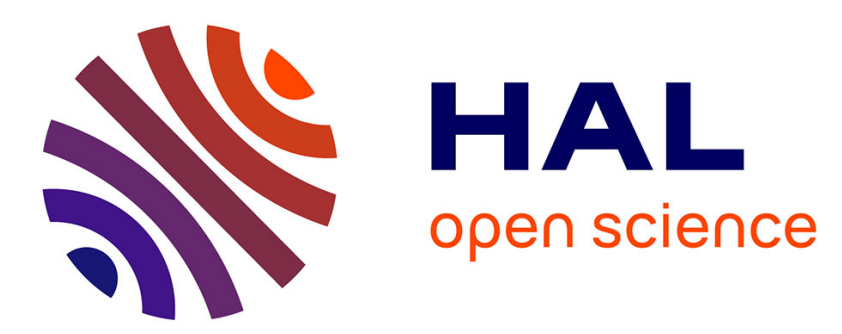

\title{
Methodological strategies in microbiome research and their explanatory implications
}

Maureen A O'Malley, Derek Skillings

\section{To cite this version:}

Maureen A O'Malley, Derek Skillings. Methodological strategies in microbiome research and their explanatory implications. Perspectives on Science, 2018, 26 (2), pp.239-265. 10.1162/POSC_a_00274 . hal-02008655

\section{HAL Id: hal-02008655 \\ https://hal.science/hal-02008655}

Submitted on 5 Feb 2019

HAL is a multi-disciplinary open access archive for the deposit and dissemination of scientific research documents, whether they are published or not. The documents may come from teaching and research institutions in France or abroad, or from public or private research centers.
L'archive ouverte pluridisciplinaire HAL, est destinée au dépôt et à la diffusion de documents scientifiques de niveau recherche, publiés ou non, émanant des établissements d'enseignement et de recherche français ou étrangers, des laboratoires publics ou privés. 


\title{
Methodological strategies in microbiome research and their explanatory implications
}

\author{
Maureen A. O'Malley \\ University of Bordeaux \\ HPS, University of Sydney, Carslaw Building, NSW 2006, Australia \\ maureen.omalley@sydney.edu.au
}

Derek J. Skillings

Immunoconcept, CNRS UMR 5164, University of Bordeaux, Bordeaux 33076, France derek.skillings@gmail.com

Keywords: Microbiota; Microbiome; Causality; Explanation; Dysbiosis

\begin{abstract}
Early microbiome research found numerous associations between microbial community patterns and host physiological states. These findings hinted at community-level explanations. 'Top-down' experiments, working with whole communities, strengthened these explanatory expectations. Now, 'bottom-up' mechanism-seeking approaches are dissecting communities to focus on specific microbes carrying out particular biochemical activities (e.g., choline metabolism pathways, Clostridium difficile suppression). To understand the interplay between methodological and explanatory scales, we examine claims of 'dysbiosis', when host illness is proposed as the consequence of a community state. Our analysis concludes with general observations about how methodologies relate to explanations, and the implications for microbiome research.
\end{abstract}

\section{Introducing microbiome research}

Microbiome research is the analysis of the aggregated molecular components of a defined microbial community ('microbiota'). ${ }^{1}$ Our examination of this field will focus on how causality is assigned in microbiome analyses, and what methodological strategies are used to identify communities or components of those communities as causal contributors to host states. Earlier microbiome research was conducted at a

\footnotetext{
${ }^{1}$ Although it was once common to distinguish the microbiota (the organisms) from the microbiome (their collective genome), current practice is often to refer to both organisms and their molecular bases as microbiomes. See Prescott (2017) for a discussion of the confused history of these terms. We will follow the older and narrower convention, but quite a lot of the literature we cite does not. The broader use of the term sometimes invokes the much older ecological meaning of 'biome' to justify the organismal application of microbiome). We will return to the potentially ecological interpretations of 'microbiome' in Section 5.
} 
broad scale via bioinformatic analyses of environmental samples, then backed up by whole community transfer experiments. These 'top-down' findings, in which the whole community is treated as a unit of investigation, indicated that communities might act as causal agents. More recently, as microbiome research has reconnected with traditional experimental methods, communities have been decomposed in the 'bottom up' search for traditional causal agents, such as single organisms, or small groups of them, and their biochemical activities. These top-down and bottom-up methodological strategies do not, however, map straightforwardly onto top-down or bottom-up explanations. This incongruity has implications for the claims that can be made about the locus of causal action.

In the following discussion, we outline some developments in how causality is investigated in microbiota-host research, and what the implications of various methodologies are for how these systems feature in explanations. We first trace how microbiome research (especially that based on human gut microbiota) has developed historically through association studies, in which community patterns have been linked to broader system outcomes such as the health and disease states of human hosts. We then discuss recent research that returns the field's focus to traditional targets of microbiological experimentation: populations and specific biochemical pathways. We illustrate this shift with brief accounts of new mechanistic insights into choline metabolism and Clostridium difficile therapy. Our analysis then turns to the explanatory conundrum of 'dysbiosis', in which claims are made about host disorders as causal products of community-scale changes in microbiota. We suggest that understanding how methodologies work at different scales can revise superficial impressions about the explanatory and conceptual implications of microbiome research.

\section{Historical background to microbiome research}

Microbiome research is a recent development in the life sciences. Its roots lie in microbiology and molecular genetics, but its implications reach beyond what is traditionally seen as microbiological subject matter. In its historically classic form, microbiology is a laboratory-based science focused on pure cultures of microorganisms. Pure culturing methods became so central to microbiology because of their experimental credentials in establishing microbial causality via Koch's postulates. The latter describe a sequence of isolating, culturing and returning microbes to hosts. They aim to demonstrate specific and consistent microorganismal effects, especially those implicated in disease and food spoilage (Gradmann 2000; Mendelsohn 2002; Ross and Woodward 2016). Biochemistry, as it rose to prominence in microbiology in the early twentieth century, further narrowed causal attributions by identifying the biochemical pathways by which single lineages of microorganisms or small groups of them produce effects (Singleton and Singleton 2016). Organism-specific activity has thus been the traditional locus of causal assignment in microbiology.

At the same time that the laboratory-based pure-culture approach became established in the late nineteenth century another set of practices developed. It focused on microorganisms in natural environments (see O'Malley 2014). This field also took a biochemical approach, and in the middle of the twentieth century productively encountered molecular genetic methods. In the 1980s, molecular efforts 
to understand microorganisms in their natural environments took a transformative turn. Norman Pace and colleagues looked beyond the genes of isolated cells in laboratories. They applied the maturing technology of DNA sequencing to in situ samples of microbial communities (e.g., Stahl et al. 1985; Olsen et al. 1986). Most of these early environmental studies focused on genes for ribosomal RNA (rRNA), which had already been established as useful markers for evolutionary analyses (Woese and Fox 1977).

As evolutionary and ecological molecular approaches gathered momentum in microbiology, sequencing technologies and bioinformatic tools were also developing in both scope and speed. Entirely new pictures began to emerge of previously unrecognized microbial diversity (e.g., Mullins et al. 1995). Culturing had for the time being reached its limits in revealing microbial diversity in many environments, often because the 'pure' growth of unknown microorganisms was obstructed by the complex interdependencies in their communal lifestyles (Amman et al. 1995). The sequencing of a very large fragment of DNA in a marine sample, followed by identification of the uncultured organismal group to which it belonged (Stein et al. 1996), was confirmation that not only was this sequence-based methodology feasible but also that it would produce novel findings. This approach made an unexpected discovery of proteorhodopsin, a light-reactive protein in marine bacteria, which was then confirmed experimentally as functionally active (Béjà et al. 2000). The proteorhodopsin study demonstrated very effectively that environmental sequencing of community samples could lead to major discoveries, and that these could then be interrogated further by classic scientific methods (see O'Malley 2008 for an overview).

With ongoing improvements in sequencing technology and large data-scale analysis, attention shifted from single genes (e.g., rRNA) to whole genomes. When these analyses targeted 'environmental' genomes (i.e., the DNA extracted from samples collected from a range of natural environments), metagenomics was born. The first use of 'metagenome' was in a manifesto for soil metagenomics in 1998 (Handelsman et al. 1998, p. R245). In that paper, molecular biologist, Jo Handelsman, and her colleagues reflected on the untapped diversity of soils, which are a major reservoir of medically and industrially important microbial compounds. The new century saw metagenomics flourishing, as DNA from a very wide range of environments and communities was sequenced and partly analysed (Handelsman 2004).

Two quite different projects spurred on the field as they showed researchers the potential of metagenomic tools. One was the metagenomic analysis of the Sargasso Sea, a huge data-collection exercise that revealed the extraordinary genetic diversity of microbial communities even in low-nutrient environments (Venter et al. 2004). The other project was the analysis of a still more restrictive environment: the acidic and anoxic runoff from a mine (Tyson et al. 2004). Identification of all the organisms in this low-diversity community was possible in the latter case; in the former, as well as assessments of population genetic structure, the function of genes (including the light-reactive proteins discovered in earlier metagenomic samples) could be tentatively assigned to uncultured organisms and inferences made about their ecological roles. 
As this new molecular field of microbial diversity studies grew, attention increasingly turned to the human body as an ecosystem in its own right. The potential of metagenomic tools was suggested for 'the second human genome' - the microorganisms untouched by the achievements of the high-profile Human Genome Project (Relman and Falkow 2001, p. 206). The gut metagenome in particular was recognized as an immense organ-like source of genes and enzymatic activities that were mostly unknown, and likely to remain so, if laboratory-based culturing studies remained the only option (e.g., Eckburg et al. 2005; Zoetendal et al. 2004). Many gut microbes can be cultured, but their full diversity (especially of anaerobes), plus their interactions, were and still are not understood in detail. To develop this understanding, international research consortia began to treat the human body as constituting an ecological niche that with the gut microbiota forms a complex multilevel system (Turnbaugh et al. 2007; O'Malley et al. 2014). This ecological perspective began to influence an expanded interpretation of the term microbiome, which was often taken now to emphasize 'biome' and its ecological connotations rather than 'ome' and its more restrictive molecular interpretation (see Footnote 1 for terminological debates).

Although non-human ecosystems are at least as interesting and important as human ones, there is no denying that the sheer amount of research on the human microbiome, and particularly the gut microbiome, make it an obvious focus for tracking how microbiome research has developed and how causal inferences have been made in that development. The vast majority of human microbiome literature focuses exclusively on bacteria, which are the most abundant forms of life in the gut. This is done not only for pragmatic reasons of reducing the complexities of analysis (i.e., leaving out viruses and other unicellular organisms), but also because the greater biochemical diversity in bacteria is thought to have more impact on the human body. But as well as sequencing the gut community genomes (or genetic markers in those genomes), then seeking patterns in that data, human microbiome projects began to explore causal claims on the basis of association studies. Causality was attributed to communities, which were initially discussed as if they were causally cohesive in the effects they brought about in hosts.

\section{Descriptions and causes: from pie-charts to mouse models}

A great deal of early research on the human gut microbiota and its microbiome produced compositional 'pie-charts' (as did microbiota studies of the skin, mouth and other niches). The charts map the DNA sequence categorizations in the microbiome of interest (Figure 1). These distributions of sequence abundances are commonly presented at the phylum level. Despite organismal groups being identified in this categorization, they are rarely characterized beyond the molecular markers that identify them. Although there is extremely fine-grained microbial diversity at the species and strain level in any single human body, it is much simpler to analyse molecular patterns at the coarse phylum level. Phyla (sometimes called 'divisions'), are a very high level of taxonomic rank, just below kingdoms, and above orders, classes, families, genera and species (in descending order of the taxonomic hierarchy). There are anywhere between 30 and a thousand bacterial phyla 
recognized by different methods and criteria. ${ }^{2}$ The great majority of healthy human guts so far sampled are dominated by only two phyla: Firmicutes and Bacteroidetes (Figure 1). Together they comprise about $90 \%$ of human microbial gut diversity.

Figure One: A variety of human microbiomes (the molecular compositions of particular communities) represented proportionally at the phylum level by piecharts (based on Spor et al. 2011; adapted by Michel Durinx (https://centimedia.org/) and used with permission from Nature/Springer/Palgrave, Nature Reviews Microbiology, copyright 2011).

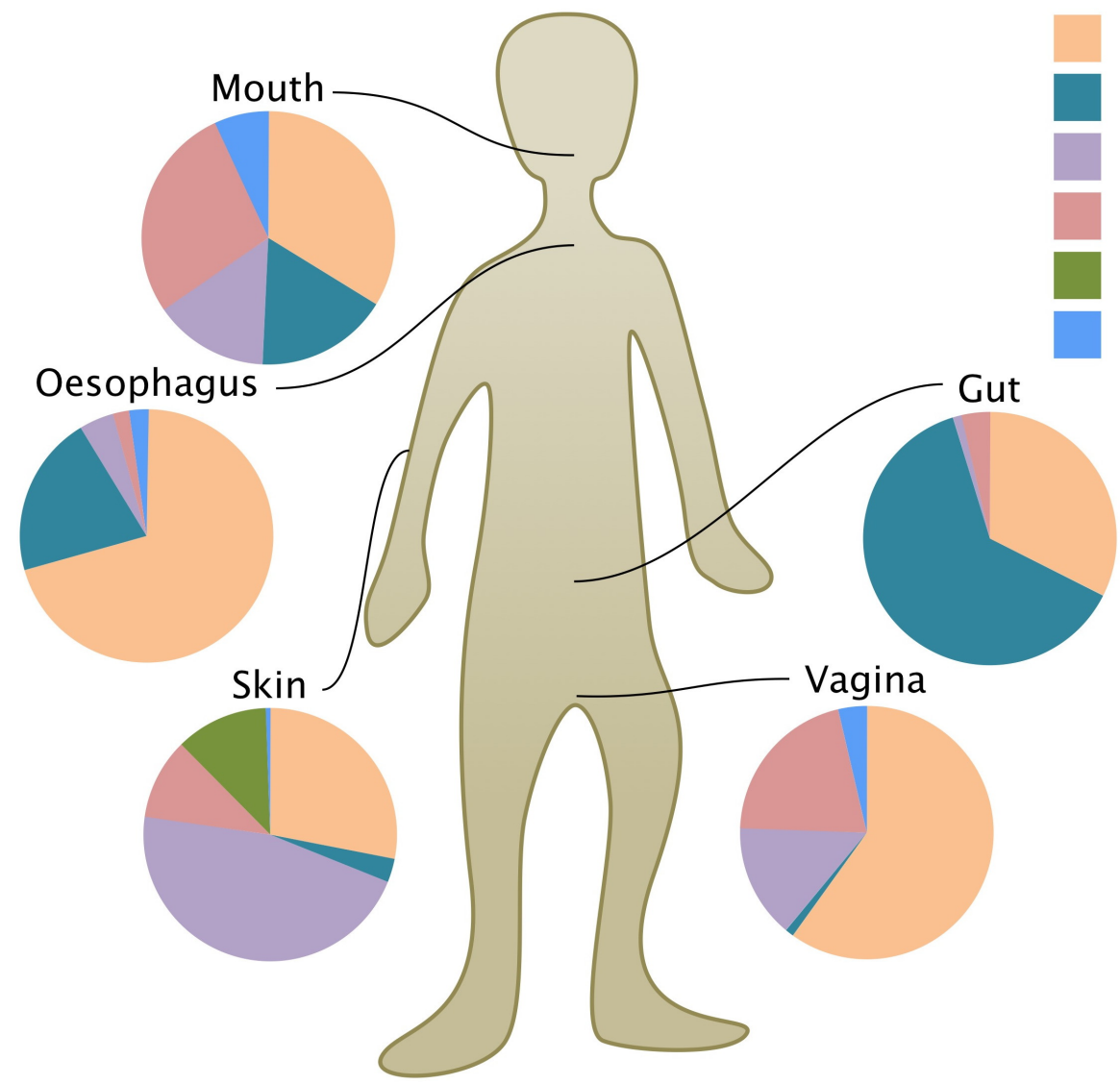

Firmicutes

Bacteriodetes

Actinobacteria

Proteobacteria

Cyanobacteria

Fusobacteria

Once the composition of a microbiome - such as that of the human gut - has been roughly described and categorized, these patterns can be 'linked' to or associated with particular health and disease states. Comparison with microbiomes in humans lacking the disease indicates visually how phyla patterns are different in diseased hosts (Figure 2). Although these comparisons are done for numerous body niches, it is the distal gut - the large intestinal colon - that has been the focus of the most work (Marchesi 2011). This trend has been driven partly by the ease of access to gut microbiota diversity, which is commonly via faecal samples. Although there are

\footnotetext{
2 The phylum rank is not recognized by official classification nomenclature (Parte 2013), and it is not clear what the boundaries for a prokaryote phylum would be, or how many bacterial phyla there might be, especially with burgeoning environmental discoveries of putative bacterial phyla (e.g., Yarza et al. 2014, Brown et al. 2015). Recent microbiome classification pushes toward lower taxonomic levels (e.g., Johnson et al. 2017; Duvallet et al. 2017), but broad-brush pictures are still the norm.
} 
questions about the adequacy of this representation, ${ }^{3}$ by and large this representativeness has been and still is taken for granted.

Figure Two: A typical representation of the human gut microbiome and associations between broad compositional patterns and disease (based on Spor et al. 2011; adapted by Michel Durinx (https://centimedia.org/) and used with permission from Nature/Springer/Palgrave, Nature Reviews Microbiology, copyright 2011).

Sometimes only a few samples underpin such patterns, which is very low-powered statistically given how much inter-individual variation exists between each human's microbiome. Ideally such associations should be interpreted as patterns worth exploring, and not as statistically robust correlations, but sometimes stronger interpretations are made.

Firmicutes

Bacteriodetes

Actinobacteria

Proteobacteria

Verrucomicrobia

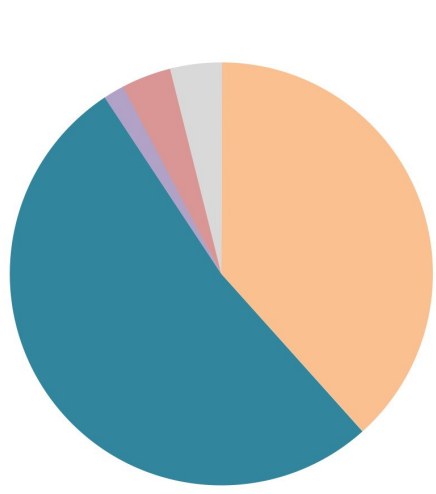

Type 2 diabetes

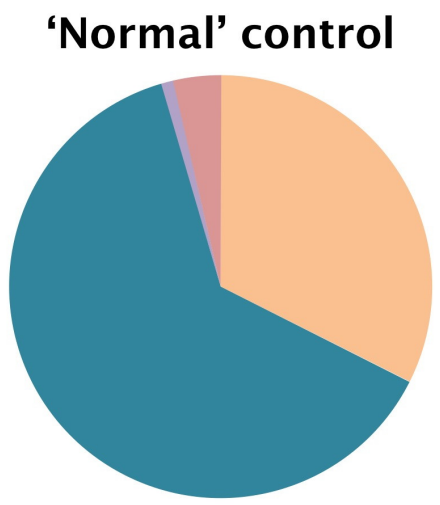

Disease cases

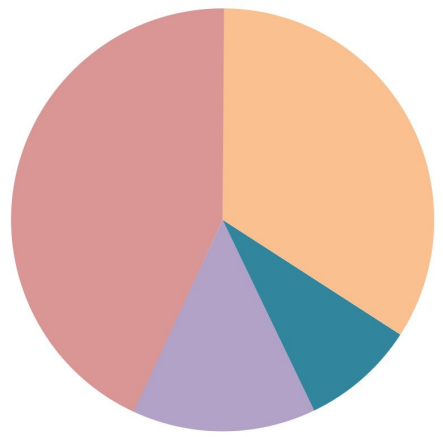

Inflammatory bowel disease

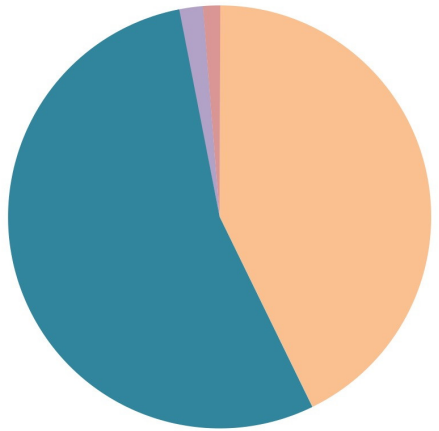

Obesity/

Metabolic syndrome

For some important diagnostic and predictive purposes, phyla proportions may potentially tell researchers about disease states. Numerous studies, including some formative microbiome research, have shown that an elevated proportion of Firmicutes is associated with host obesity and the metabolic syndrome accompanying it (e.g., Ley et al. 2006, Turnbaugh et al. 2006, 2008). Phylum-level patterns associated with conditions such as human obesity have been described as

\footnotetext{
${ }^{3}$ The composition of microbiomes sampled via faeces versus intestinal biopsies are often not concordant (Momozawa et al. 2011). There are indications that samples taken directly from the gut will more accurately predict disease states (Gevers et al. 2014).
} 
'highly conserved bacterial traits' that affect host phenotype (Ley et al. 2006:1023). Although obesity investigations are emblematic of human microbiome association studies, a wide range of other diseases, including brain and behavioural disorders (Bercik et al. 2011; Hsiao et al. 2013), have now been associated with coarse changes in microbiota diversity. Many of these association studies do not, however, go on to examine any causal implications very thoroughly (i.e., whether changes in composition are causes or effects of disease states).

Knowledge of associations can in some cases be backed up by experimental regimes, in which entire microbiota are transferred into animal hosts, mostly mouse models. Led by early efforts in Jeffrey Gordon's lab, microbiome researchers began to manipulate microbiota experimentally. This work involves 'germ free' laboratory mice. They are born by caesarian section and raised in sterile environments (including irradiated food) so that they are not colonized by microbiota, unlike conventionally born and reared mice. Microbiome researchers transplant microbiota samples - commonly via faeces from conventional mice with specific phenotypic properties, and even, surprisingly, from humans ${ }^{4}$ - into these germ-free model mammals, and then track physiological changes in order to scrutinize cause-effect relationships (Turnbaugh et al. 2006; 2009; Ridaura et al. 2013). Researchers have frequently found that transplanting phyla-differentiated microbiota into mice can make a major physiological difference to the host. In other words, transmission of phenotype at the organismal (mouse) level can occur via transmission of the microbiota. In these cases, 'the microbiome is considered causal' (Goodrich et al. 2014, p. 250).

Although there are additional and possibly confounding causal factors that must be taken into consideration (especially diet, and medications such as antibiotics), research using mouse models and entire microbiota transplants became the gold standard for causal attributions in microbiome studies. Once again, obesity has been a particularly successful example of phenotypic transfer by microbiota transplant, confirming the causality inferred from bioinformatic associations (e.g., Turnbaugh et al. 2009). Firmicutes-Bacteroidetes proportions, or other broad changes in community diversity, continued to be implicated as significant causal contributors to obesity and other diseases.

But as microbiome research has developed further, phylum-level proportionality or decreased diversity turns out to offer less insight into human (and mouse) obesity and other conditions than originally anticipated (Duvallet et al. 2017; Sze and Schloss 2016; Finucane et al. 2014, Walters et al. 2014). ${ }^{5}$ Both increases and decreases of key phyla may be associated with obesity. Even worse, experimental replications have contradicted previously postulated effects of microbiota changes (Harley and Karp 2012; Schwiertz et al. 2010; Fleissner et al. 2010). One response is to see if consistent observations can be achieved by increasing sample size (e.g., Falony et al. 2016; Beaumont et al. 2016). Another is to look within the community for smaller scale causal agents.

\footnotetext{
${ }^{4}$ This is surprising because of the phylogenetic specificity of many microbiota-host relationships (Chung et al. 2012; however, cf. Seedorf et al. 2014, which shows some unexpected flexibility).

${ }^{5}$ Small sample sizes and their statistically underpowered findings play a role here, as noted in Section 2.
} 


\section{From top-down to bottom-up microbiome analyses}

Establishing fine-grained causal relationships has become for many microbiome researchers the driving aim of the field (Surana and Kasper 2017; Fischbach and Segre 2016; Gilbert et al. 2016). This wave of research focuses on pinpointing, for example, 'the specific bacteria exclusively associated with obesity' and other healthdisturbing syndromes (Zhang et al. 2009:2365; Zhao 2013; Harley and Karp 2012).

Some researchers describe efforts to carry out finer grained experimentalmechanistic work as 'bottom-up' microbiome research, in order to distinguish it from the 'top-down' methodologies discussed in Section 3. The latter are driven by computational analyses and pattern-seeking approaches, then supplemented by whole-microbiota transplants to mice (e.g., Huttenhower et al. 2014; Macpherson et al. 2015; Moya and Ferrer 2016). ${ }^{6}$ However, many bottom-up approaches are based on or include top-down approaches that treat the community as a whole before doing traditional cause-establishing research. New microbiome research on choline metabolism $^{7}$ and Clostridium difficile therapy show how these emerging decompositional approaches work.

\subsection{Choline metabolism}

Choline is an essential human dietary nutrient found primarily in red meat and egg yolks. Gut microbes convert some of it anaerobically to trimethylamine, which is then oxidized in the liver. Trimethylamine in this oxidized form is associated with cardiovascular problems in humans and mice (Tang and Hazen 2014). ${ }^{8}$ An important step in unravelling this causal chain showed that mice fed with choline tend to develop atherosclerosis (hardening of the arteries), and more precisely, that suppressing all gut microbes in mice inhibits this disease (Wang et al., 2011). But rather than attribute causal agency to the microbiota generally or to certain proportions of phyla, the next research step zoomed in on a gene cluster of a sulphate-reducing gut bacterium. This organism could be cultured and manipulated genetically (Cracium and Balskus 2012). Researchers then went on to characterize the exact mechanism of how this bacterium metabolizes choline. They noted that their discovery 'shows the potential of combining analysis of a biochemical mechanism with bioinformatic analysis' (Cracium and Balskus 2012, p. 21310), so that specific causal agents can be localized in broader microbiome patterns. This combination of top-down and bottom-up methodologies also worked for studies that tracked the conversion of another red meat substance, $L$-carnitine, to trimethylamine via gut microbes (see Falony et al. 2015 for an overview).

What appears to be happening in the choline research area is a traditional decompositional analysis, in which microbiota systems are broken down to their parts, and methods applied to identify the details and ordering of causal effects (for a list of other examples, see Fischbach and Segre 2016). This classic approach aims

\footnotetext{
${ }^{6}$ Not all these citations refer to exactly the same combinations of approach, but their distinctions are broadly consistent. We include the mouse microbiota transplants in the topdown category, because these experiments are transferring the whole community and looking for host-wide effects.

${ }^{7}$ Thanks to Andrew Roger (Dalhousie) for suggesting this example.

${ }^{8} \mathrm{~A}$ few studies show no connection between choline intake and atherosclerosis, but they are in the minority.
} 
to fill in the mechanistic links in a causal chain. Other interactions exist, to be sure, but they are given background status. For example, when the choline metabolism process is tracked by experiment and biochemical inference to identify a pathway in an organism, it does not mean that the production of trimethylamine is necessarily confined to a single organism (see Zhu et al. 2016) but that any additional choline metabolizers need to be studied separately.

Other areas of research, particularly concerned with how the human immune system develops in concert with microbiota composition and function, also show considerable promise for filling in detailed mechanistic steps between microbiota patterns and human disease (Round and Mazmanian 2009; Hooper et al. 2012). Both biochemistry and immunology are experimentally focused sciences, and they appear to be drawing on large-scale bioinformatic analyses primarily in order to stimulate hypotheses and their experimental investigation. ${ }^{9}$ But not all microbiota research works in this way; some of it shows consistent effects from community-level intervention. Nevertheless, even in these cases, there is explanatory pressure to 'downsize' causality by pinning causal interactions to the organismal level.

\subsection{Clostridium difficile therapy}

One of the most dramatic success stories in microbiome research comes from the use of faecal microbiota transplants (FMTs) as treatments for Clostridium difficile infections in human intestines. This organism, which is often carried asymptomatically and only becomes pathogenic after antibiotic treatments (especially post-surgery), can cause long-term illness, pain and eventually death to infected humans. $C$. difficile forms spores, which enable transmission and also resistance to antibiotic therapies against the organism. More than four decades of studies of various degrees of rigour and duration have shown that microbiota transplants from healthy humans can suppress $C$. difficile activities and effects (Kassam et al. 2013; van Nood et al. 2013). The relative proportion of Firmicutes and Bacteroidetes is reportedly 'restored or 'rebalanced' in the receiver's gut by microbiota transplants, and the various symptoms of $C$. difficile infection disappear or ameliorate as this general state of 'dysbiosis' -an unhealthy composition of the community - is remedied (Borody and Khoruts 2012).

However, even though this intervention is done at a community scale (i.e., the entire microbiota, or at least as many as survive passage from one human body to another via faecal transplant), for some scientists this strategy is seen as a stop-gap measure rather than an intervention that suggests causal efficacy at the community level. There has already been some success in decomposing the 'anti-dysbiotic' FMT microbiota, experimentally and mathematically by 'rational design.' This work targets the precise causal agents that ameliorate $C$. difficile infections (e.g., Buffie et al. 2015; Lawley et al. 2012, Almeida et al. 2016). In a particularly powerful combination of methods - biochemical experiments, bioinformatic analysis and mathematical modelling - one of these studies identified the secondary bile acid synthesis carried out by certain organisms (other Clostridium species, especially $C$. scindens) as the specific biochemical mechanism by which $C$. difficile growth is suppressed (Buffie et al. 2015).

\footnotetext{
${ }^{9}$ This combination of top-down and bottom-up methods is why the proteorhodopsin story was such a powerful motivator for metagenomic research (see Section 2).
} 
The identification of finer grained causality in bottom-up studies is done not just for the sake of basic scientific explanation, but also to develop therapeutic treatments that exploit these discoveries of causal pathways. Despite the successes of community-level FMT interventions on $C$. difficile infections, drug development efforts are based on the belief that causality should be attributable to identifiable lineages, and to isolatable pathways in those lineages. These 'bottom-up' accounts of causal agency in microbiota research thus attempt to follow quite traditional scientific practices in microbiology, even when initial efforts were able to make loose causal ascriptions at the higher community level.

\section{Dysbiosis and homeostasis in microbiome explanations}

Constructing causal explanations is a key motivating activity for scientists. Descriptions of these causes are part of the standard mechanistic explanatory apparatus that is taken for granted as the basis of good science. ${ }^{10}$ Decompositional analyses are usually what enable mechanistic causal attributions (Bechtel and Richardson 1993). Retaining a causal focus on communities might thus seem unlikely to continue as the field develops. However, the microbiome story is not necessarily one of a broad-brush view being rightfully replaced by a more detailed and complete one as the field develops. Microbiome researchers continue to hint at or even argue explicitly for community-level causation that might resist such decomposition.

One of the most obvious ways this occurs is when microbiome researchers attribute 'dysbiosis' to the community level, by reasoning that 'some diseases might result from dysbiosis rather than the presence of a single disease-causing microbe' (Clemente et al. 2012, p. 1263). Such claims are justified by beliefs that it is likely that no one single organism will work most effectively, but rather a complex assortment of organisms will provide the maximum benefit' to the host (Petersen and Round 2014:1030). Dysbiosis in microbiome research is defined very loosely, and refers broadly to a "compositional or functional shift within host-associated microbial communities that has the potential to facilitate growth of pathogens and/or [the] onset [of] diseases' (Arnold et al. 2016, p. 889; see Hooks and O'Malley 2017 for a critique).

Changes to phylum ratios or general decreases in diversity commonly serve as markers of dysbiotic alterations (Lewis et al. 2015). These changes are detected post-hoc, when hosts known to be diseased exhibit 'change to the composition of resident commensal communities relative to the community found in healthy individuals' (Petersen and Round 2014, p. 1024). However, this ideal or normal state is usually unspecified, or simply described as having more diversity. ${ }^{11}$ Moreover, it is usually impossible to discern whether inferred dysbioses occur prior to the disease they are putatively causing or afterward, as a consequence of the disease (Bäckhed

\footnotetext{
${ }^{10}$ The same is not true of much medical research, especially 'evidence-based medicine', where evidence for mechanisms is often very low down in the evidence hierarchy (Andersen 2012; Howick 2011; La Caze 2011).

${ }^{11}$ See, however, Gevers et al. (2014), for an example of a more precise 'dysbiosis index' correlated with a particular disease state.
} 
et al. 2012). There may also be common causes, such as diet or inflammation, that produce both the altered microbiota composition and the disease.

When dysbiosis is proposed as a system-level explanation of host physiology, it is often implied that there is a 'balanced' state to which the whole microbiota normally contributes. This balance is thought to arise from the evolved normalness or optimality of the sum of interactions between human bodies and their microbial commensals (e.g., Belkaid and Hand 2014; Fuentes et al. 2014, Reid et al. 2011). It is common in microbiota research to refer to this supposedly balanced state as one of 'homeostasis', although sometimes the terms 'eubiosis' and 'normobiosis' are used (e.g., LePage et al. 2013; Schulberg and De Cruz 2016). Just as for claims about dysbiosis, homeostasis is asserted very casually and loosely, as something 'normal' and physiologically desirable (e.g., Reid et al. 2011). Quantified theoretical claims, such as might accompany traditional cybernetic views about homeostasis, are not attempted.

What do bottom-up methodological developments in microbiome research mean for dysbiosis and homeostasis and their implicit and explicit claims about communitylevel causation? And more generally, why might changes in community-scale composition, especially different proportions of phyla, even be thought of as plausible causal agents in the first place? One answer comes from general perspectives on systems and explanations at the system level. It is well known that system states can produce effects, and that such relationships may not be revealed by simple experimentation that searches for and establishes single mechanisms and linear causal chains (see Green et al. 2017).

A more substantive reason for continuing to focus on the community as a causal locus is the extraordinary functional redundancy in communities such as those of the human gut. Knock out one species or strain, and another will commonly supply the same products. Robust function supplied by networks of genes and metabolic pathways may be the relevant explanatory locus rather than the more transient individual taxonomic units that bear and share such genes (Doolittle and Booth 2016; Taxis et al. 2015; Louca et al. 2016). These networks will often not be localized in single populations of organisms. Functional accounts of how communities robustly bring about health or disease are now seen as a way forward for community-based explanations of host state (Knights et al. 2013; Moya and Ferrer 2016; Levy et al. 2017). Nevertheless, to understand these functional contributions, decompositional analysis of the community still has to be done to show how members work together to create functions that are distributed across different lineages of organisms. Even when clusters of organisms from different lineages are known to be causal players, knowledge of the individual genes and pathways that make up that cluster is required by the current methodological configuration and explanatory expectations of the field.

For example, network models based on high-throughput data (which microbiome research has in abundance) are an important means of making system-level explanations (Leyeghifard et al. 2017; Greenblum et al. 2013, Borenstein 2012; Faust and Raes 2012). However, revealing the organizational structures of microbiome networks continues to be done on the basis of associations (e.g., Greenblum et al. 2012), and many network explanations still require the identification of localized mechanisms (e.g., Noecker et al. 2016). A different strategy is to analyse community- 
scale correlations with host states more rigorously in order to evaluate whether causal claims can be justified (Gilbert et al. 2015; Cho and Blaser 2012). Larger samples and more detailed analysis of the conditions that affect microbiota composition will also help reframe and refine community association approaches (Falony et al. 2016), and thus potentially enable system-level explanations of microbiome effects.

Many microbiome researchers also expect that community-scale ecological models will eventually enable predictions and explanations of microbiota states and their effects (Costello et al. 2012; Marino et al. 2014; Coyte et al. 2015). In plant and animal ecology, models of community stability have been important for explanations of ecosystem dynamics that range from collapse to flourishing (see McCann 2000; Justus 2008). Likewise, in microbiome research, the stability or instability of wholecommunity composition is thought to be an explanatory candidate for some disease states in hosts (e.g., Jeffery et al. 2016). Community genetics, usually applied to plant communities (Whitham et al. 2006), ${ }^{12}$ may also provide explanatory resources for microbiome explanations at the community scale by showing how genetic variation in populations contributes to community structure and ecosystem behaviour (Skillings 2016).

An illustration of a rudimentary ecological explanation of microbiota and host builds on the success of FMTs in 'curing' $C$. difficile pathologies. The influx of donor microbes is hypothesized to affect the niche structure of the gut due to the transplanted microorganisms taking over niches that $C$. difficile had usurped. This takeover also explains the diversity depletion of the pre-transfer microbiota, which is strongly associated with C. difficile infection (Khoruts et al. 2010; Lawley and Walker 2012). Explanations like this, while still sketches, could be fleshed out and provide genuinely community-level ecological explanations. This fleshing-out, however, would require the initial decomposition of the community to understand the functional roles, niches and interactions that led to the success of the transplant.

Although we think it likely that the future of microbiome explanation will be ecological (and would thus emphasize the 'biome' interpretation of microbiomes - see Section 1 ), a great deal of methodological development and detailed research is required before community-level hypotheses about stability, robustness and 'dysbiosis' are established on a stronger evidential and inferential knowledge base. For the time being, such hypotheses provide at best indications of scenarios that need further attention and might eventually be filled in mechanistically. A key issue here is whether communities treated as methodological targets will map onto the explanatory targets of microbiome research.

\section{Implications of microbiome research methods for explanation}

Earlier, we described how microbiology's pure-culture approaches had to isolate organisms from their complex communities in order to home in on central relationships of cause and effect. Now, microbiome research is in search of explanations generated on the basis of findings from community-level methods. It is not clear that current methodologies are necessarily going to achieve explanations at

\footnotetext{
${ }^{12}$ This form of community genetics is not the medical or public health version.
} 
the community scale (Table 1). Current restrictions on transforming community-level findings to explanations at the same scale do not mean top-down attributions are irrelevant or redundant: at the very least top-down associations and experiments play a central role in identifying broad and potentially causal relationships. But it is more traditional methods of decomposing those entities and isolating very specific effects that seem to be picking up the epistemic baton for the microbiome research community.

Table 1: Top-down and bottom-up interpretations. Although it looks as if the entries in each column should work together, we suggest that microbiome research may currently be restricted to explanations on the bottom-up side, even when top-down methodologies are employed. Current methodological and explanatory achievements are shaded.

\begin{tabular}{|l|l|l|}
\hline & Top-down & Bottom-up \\
\hline Methodological target & Community patterns & $\begin{array}{l}\text { Individual organisms } \\
\text { and pathways }\end{array}$ \\
\hline Explanatory level & $\begin{array}{l}\text { System states as } \\
\text { mechanisms }\end{array}$ & $\begin{array}{l}\text { Single components and } \\
\text { mechanisms }\end{array}$ \\
\hline Explanatory strategy & Non-decompositional & Decompositional \\
\hline
\end{tabular}

Early microbiome research, driven by bioinformatic analyses and top-down experimentation, necessarily worked with and drew attention to community-scale patterns (Manor et al. 2014; Huss 2014). But now, as more traditional methods of experimentation are brought to bear on microbiota, the causal focus often becomes a smaller system: an organism or population of organisms that possesses particular biochemical pathways. For better or worse, scientific confidence about causal claims tends to be built on experimental manipulation of small-scale systems and their components (Craver 2006). ${ }^{13}$

Claims about dysbiosis might at first glance be thought to function as explanatory place-holders for more substantive accounts of how community proportions or diversity generally affect host health. Particularly when causality is localized to specific microbe-host interactions, as in the choline and $C$. difficile cases, suggestions of dysbiosis as the loss of homeostasis or natural balance appear to be too loose and global to do much genuine explanatory work. Relationships between microorganisms and their human hosts might in some cases at least be explained by specific interactions between individual organisms and pathways, rather than broad assertions about dysbiosis and homeostasis. This does not mean that claims about community-level states such as dysbiosis are made false, but that the pressure is mounting for them to be evaluated more rigorously and filled in mechanistically (e.g., Olesen and Alm 2016; Hooks and O'Malley 2017).

\footnotetext{
${ }^{13}$ In biomedical research, where microbiome analysis has achieved considerable infiltration, confidence comes from randomized controlled trials. Such trials are not anticipated to play a large role in microbiome research's immediate future, except for simple treatment regimes involving probiotics and microbiota transplants.
} 
However, although mechanism-oriented research can indeed provide causal explanations of particular phenomena, it is clear there are many instances when causal complexity overwhelms straightforward mechanistic accounts (Green et al. 2017; Andersen 2012). Even though microbial communities may eventually be decomposed causally, explanatory payoff is still expected at the larger scale, on the grounds that network or large-scale ecosystem properties may achieve host effects. However, achieving community-level explanations of host health and disease continues to be dependent on knowledge about small-scale causal agents. Assigning causality in such situations and pursuing broad system-level mechanisms (e.g., putative dysbiosis states) still await the development and application of appropriate methodologies and explanatory strategies in microbiome research. So far, the maturation of microbiome research highlights how explanations are not necessarily generated at the same scale as the methodological starting point.

\section{Implications of microbiome explanations for conceptualizing communities}

One important consequence of understanding the relationship between methodological and explanatory strategies in microbiome research has to do with the field's implications for biological and evolutionary individuality (this is a topic that commentators from outside the field have found particularly interesting, although many of them have paid limited attention to microbiome research practices). If microbial communities bring about host effects only as a whole, then these systems might be understood as closely integrated, functionally intertwined, mutually dependent groups of organisms. Often, claims that microbial communities form cohesive causal entities flow into assumptions of evolutionary unity; such assumptions are now seriously criticized (e.g., Douglas and Werren 2016). ${ }^{14}$ However, even when it is only physiological unity being considered, the degree of cohesiveness between members in any community still needs to be worked out with regard to specific causal interactions. Many important negative and positive causal interactions will almost inevitably localize to subpopulations within those communities, and vary according to how those groups interact differentially with other populations in the host environment (e.g., Estrela et al. 2016).

The strength of any interactions between populations is also dependent on environmental variables such as diet, dispersal ability, geography, and host physiology (Falony et al. 2016). These factors are unlikely to operate on different populations in exactly the same way, nor on the relations between them. There will be different degrees of cohesiveness between components (populations) of the microbiota. Thus, for many explanatory purposes, such as the investigation of specific health or illness states, the focus will not be the community as a whole. Each case will require careful analysis of specific causal interactions, and assessments of whether microbiota activities are widespread or localized, negative or positive. There may be some historical parallels here with plant community research of the early part of the twentieth century. Notions of a highly organized community (a physiological

\footnotetext{
${ }^{14}$ We will not linger over these debates here, but do suggest they need viewing from an explanatory angle.
} 
unit) were eventually eclipsed by views that such entities are more helpfully understood as structured assemblies of individual organisms with specific causal inputs (Odenbaugh 2007). By understanding these individual activities, insight into community dynamics is gained.

One factor that may have contributed to assumptions about the physiological unity of microbiota is that early human microbiome analyses took for granted the mutual benefits of human-microbiota relationships (e.g., Hooper and Gordon 2001; Bäckhed et al. 2005). Evolutionary accounts have been given of these assumed mutual benefits. For example,

'The shared evolutionary fate of humans and their symbiotic bacteria has selected for mutualistic interactions that are essential for human health, and ecological or genetic changes that uncouple this shared fate can result in disease' (Dethlefsen et al. 2007, p. 811).

The underlying rationale is that because humans without microbes do not exist, evolution must surely have worked out ways for humans to get along positively, and even optimally, with their passengers. There should, therefore, be causally tight and ultimately beneficial relationships that unify host and microbiota. This line of thinking informs views of dysbiosis: perturb the evolved mutualistic relationship, and there will be bad consequences for the whole system of host and microbiota (e.g., Wu and Lewis 2013; Schwabe and Jobin 2013).

But as microbiota relationships are analysed more closely both theoretically and experimentally, it is clear that ongoing relationships between host and microbiota are very likely to be antagonistic, competitive and exploitative rather than cooperative (Coyte et al. 2015). The balance-sheet of cooperative and competitive relationships will vary over time and circumstance. Calibrating these relationships requires knowledge about individual lineages, their functions, and how they interact (e.g., Rakoff-Nahoum et al. 2016). This ecological focus then informs evolutionary accounts of the community. Even long-term evolutionary associations between microbes and human hosts (plus other mammals) are limited to a few microbial lineages (Grouissin et al. 2017), thus limiting what can be said about the microbiota as a whole causal unit with selected effects on the host.

\section{In summary}

Focusing on the interaction between methodology and explanation in microbiome research may thus deflate expectations about groups of diverse organisms (such as hosts and their microbial communities) as unified biological or evolutionary entities. Identifying the actual locus of explanation is central to understanding not just what microbiota are but what they do. As we have argued, there are many ways in which explanation may be pitched at the system level. Genuinely ecological approaches to explanation can make sense of phenomena produced by communities through a range of interactions, many of which have yet to be understood at the relevant causal level. But for now, disentangling explanatory expectations from methodological achievements can help to understand what the field of microbiome research has accomplished and where it might potentially develop. 


\section{Acknowledgements}

We are grateful for comments from discussion groups at Dalhousie University and the University of Sydney, as well as from one of our anonymous referees. MAO is supported by the French government's 'Investments for the Future' Programme, IdEx Bordeaux (ANR-10-IDEX-03-02). DJS is funded by the European Research Council (ERC) under the European Union's Horizon 2020 research and innovation programme, Grant agreement no 637647 (IDEM).

\section{References}

Almeida, Rowena, Teklu Gerbaba, and Elaine O. Petrof. 2016. "Recurrent Clostridium difficile Infection and the Microbiome." Journal of Gastroenterology 51:110.

Amman, Rudolf I., Wolfgang Ludwig, and Karl-Heinz Schleifer. 1995. "Phylogenetic Identification and In Situ Detection of Individual Microbial Cells Without Cultivation." Microbiology Reviews 59:143-169.

Andersen, Holly. 2012. "Mechanisms: What Are They Evidence for in EvidenceBased Medicine?" Journal of Evaluation in Clinical Practice 18:992-999.

Arnold, Jason W., Jeffrey Roach, and M. Andrea Azcarate-Peril. 2016. "Emerging Technologies for Gut Microbiome Research." Trends in Microbiology 24:887-901.

Bäckhed, Frederik, Claire M. Fraser, Yehuda Ringle, Mary Ellen Sanders, R. Balfour Sartor, et al. 2012. "Defining a Healthy Human Gut Microbiome: Current Concepts, Future Directions, and Clinical Applications." Cell Host \& Microbe 12:611-622.

Bäckhed, Frederik, Ruth E. Ley, Justin L. Sonnenburg, Daniel. A. Peterson, and Jeffrey I. Gordon, J. I. (2005). "Host-Bacterial Mutualism in the Human Intestine." Science 307:1915-1920.

Beaumont, Michelle, Julia K. Goodrich, Matthew A. Jackson, Idil Yet, Emily R. Davenport, et al. 2016. "Heritable Components of the Human Fecal Microbiome are Associated with Visceral Fat." Genome Biology 17:189.

Bechtel, William, and Robert C. Richardson. 1993. Discovering Complexity: Decomposition and Localization as Strategies in Scientific Research. Princeton NJ: Princeton University Press.

Béjà, Oded, L. Aravind, Eugene V. Koonin, Marcelino T. Suzuki, Andrew Hadd, et al. 2000. "Bacterial Rhodopsin: Evidence for a New Type of Phototrophy in the Sea."

Science 289:1902-1905.

Belkaid, Yasmin, and Timothy W. Hand. 2014. "Role of the Microbiota in Immunity and Inflammation." Cell 157:121-141.

Bercik, Premysl, Emmanuel Denou, Josh Collins, Wendy Jackson, Jun Lu, et al. 2011. "The Intestinal Microbiota Affect Central Levels of Brain-Derived Neurotropic Factor and Behavior in Mice." Gastroenterology 141:599-609.e3. 
Borenstein, Elhanan. 2012. "Computational Systems Biology and In Silico Modeling of the Human Microbiome." Briefings in Bioinformatics 13:769-780.

Borody, Thomas J., and Alexander Khoruts. 2012. "Fecal Microbiota Transplantation and Emerging Applications." Nature Reviews Gastroenterology 9:88-96.

Brown, Christopher T., Laura A. Hug, Brian C. Thomas, Itai Sharon, Cindy J. Castelle, et al. 2015. "Unusual Biology Across a Group Comprising More Than 15\% of Domain Bacteria." Nature 523:208-211.

Buffie, Charlie G., Vanni Bucci, Richard R. Stein, Peter T. McKenney, Lilian Ling, et al. 2015. "Precision Microbiome Reconstitution Restores Bile Acid Mediated Resistance to Clostridium difficile." Nature 205:205-208.

Cho, Ilseung, and Martin J. Blaser. 2012. "The Human Microbiome: At the Interface of Health and Disease." Nature Reviews Genetics 13:260-270.

Chung, Hachung, Sünje J. Pamp, Jonathan A. Hill, Neeraj K. Surana, Sanna M. Edelman, et al. 2012. "Gut Immune Maturation Depends on Colonization with a HostSpecific Microbiota.” Cell 149:1578-1593.

Clemente, Jose C., Luke K. Ursell, Laura Wegener Parfrey, and Rob Knight. 2012. "The Impact of the Gut Microbiota on Human Health: An Integrative View." Cell 148:1258-1270.

Costello, Elizabeth K., Keaton Stagaman, Les Dethlefsen, Brendan J. M. Bohannan, and David A. Relman. 2012. "The application of ecological theory toward an understanding of the human microbiome." Science 336:1255-1262.

Coyte, Katherine Z., Jonas Schluter, and Kevin R. Foster. 2015. "The Ecology of the Microbiome: Networks, Competition, and Stability." Science 350:663-666.

Craciun, Smaranda, and Emily P. Balskus. 2012. "Microbial Conversion of Choline to Trimethylamine Requires a Glycyl Radical Enzyme." Proceedings of the National Academy of Sciences U.S.A. 109:21307-21312.

Craver, Carl F. 2006. "When Mechanistic Models Explain.” Synthese 153:355-376.

Dethlefsen, Les, Margaret McFall-Ngai, and David A. Relman. 2007. An ecological and evolutionary perspective on human-microbe mutualism and disease. Nature 449:811-818.

Doolittle, W. Ford, and Austin Booth. 2016. "It's the Song, Not the Singer: An Exploration of Holobiosis and Evolutionary Theory." Biology and Philosophy 32:5-24.

Douglas, Angela E., and John H. Werren. 2016. "Holes in the Hologenome: Why Host-Microbe Symbioses Are Not Holobionts." mBio 7(2):e02099-15. 
Duvallet, Claire, Sean M. Gibbons, Thomas Gurry, Rafael. A. Irizarry, and Eric Alm. 2017. "Meta-Analysis of Gut Microbiome Studies Identifies Disease-Specific and Shared Responses." Nature Communications 8:1784.

Eckburg, Paul B., Elisabeth M. Bik, Charles N. Bernstein, Elizabeth Purdom, Les Dethlefsen, et al. 2005. "Diversity of the Human Intestinal Microbial Flora." Science 308:1635-1638.

Estrela, Sylvie, Benjamin Kerr, and J. Jeffrey Morris. 2016. "Transitions in Individuality Through Symbiosis." Current Opinion in Microbiology 31:191-198.

Falony, Gwen, Sara Vieira-Silva, and Jeroen Raes. 2015. "Microbiology Meets Big Data: The Case of Gut Microbiota-Derived Trimethylamine." Annual Review of Microbiology 6:305-321.

Falony, Gwen, Marie Joossens, Sara Viera-Silva, Jun Wang, Youssef Darzi, et al. 2016. "Population-Level Analysis of Gut Microbiome Variation." Science 352:560564.

Faust, Karoline, and Jeroen Raes. 2012. "Microbial Interactions: From Networks to Models." Nature Reviews Microbiology 10:538-550.

Finucane, Mariel M., Thomas J. Sharpton, Timothy J. Laurent, and Katherine S. Pollard. 2014. "A Taxonomic Signature of Obesity in the Microbiome? Getting to the Guts of the Matter." PLOS One 9(1):e84689.

Fischbach, Michael A., and Julia A. Segre. 2016. "Signaling in Host-Associated Microbial Communities." Cell 164:1288-1300.

Fleissner, Christine K., Huebel, Nora, Mohamed M. A. El-Bary, Gunnar Loh, Susanne Klaus, and Michael Blaut. 2010. "Absence of Intestinal Microbiota Does Not Protect Mice From Diet-Induced Obesity.” British Journal of Nutrition 104:919-929.

Fuentes, Susana, Els van Nood, Sebastian Tims, Ineke H.-D. Jong, Cajo J. F. ter Braak, et al. 2014. "Reset of a Critically Disturbed Microbial Ecosystem: Faecal Transplant in Recurrent Clostridium difficile Infection." ISME Journal 8:1621-1633.

Gevers, Dirk, Subra Kugathasan, Lee A. Denson, Yoshiki Vázquez-Baeza, Will Van Treuren, Boyu Ren, et al. 2014. "The Treatment-Naïve Microbiome in New-Onset Crohn's Disease.” Cell Host \& Microbe 15:382-392.

Gilbert, Jack A., Robert A. Quinn, Justine Debelius, Zhenjiang Z. Xu, James Morton, et al. 2016. "Microbiome-Wide Association Studies Link Dynamic Microbial Consortia to Disease." Nature 535:94-103.

Goodrich, Julia K., Sara C. Di Rienzi, Angela C. Poole, Omry Koren, William A. Walters, et al. 2014. "Conducting a Microbome Study." Cell 158:250-262. 
Gradmann, Christoph. 2000. "Isolation, Contamination, And Pure Culture:

Monomorphism and Polymorphism of Pathogenic Micro-Organisms as Research Problem 1860-1880." Perspectives on Science 9:147-172.

Green, Sara, Maria Şerban, Raphael Scholl, Nicholaos Jones, Ingo Brigandt, and William Bechtel. 2017. "Network Analyses in Systems Biology: New Strategies for Dealing with Biological Complexity." Synthese doi:10.1007/s11229-016-1307-6.

Greenblum, Sharon, Hsuan-Chao Chiu, Roie Levy, Rogan Carr, and Elhanan Borenstein. 2013. "Towards a Predictive Systems-Level Model of the Human Microbiome: Progress, Challenges, and Opportunities." Current Opinion in Biotechnology 24:810-820.

Greenblum, Sharon, Peter J. Turnbaugh, and Elhanan Borenstein. 2012. "Metagenomic Systems Biology of the Human Gut Reveals Topological Shifts Associated with Obesity and Inflammatory Bowel Disease." Proceedings of the National Academy of Sciences USA 109:594-599.

Grouissin, Mathieu, Florent Mazel, Jon G. Sanders, Chris S. Smillie, Sébastian Lavergne, et al. 2017. "Unraveling the Processes Shaping Mammalian Gut Microbiomes over Evolutionary Time." Nature Communications 8:14319.

Handelsman, Jo. 2004. "Metagenomics: Application of Genomics to Uncultured Microorganisms." Microbiology and Molecular Biology Reviews 68:669-685.

Handelsman, Jo, Michelle R. Rondon, Sean F. Brady, Jon Clardy, and Robert M. Goodman. 1998. "Molecular Biological Access to the Chemistry of Unknown Soil Microbes: A New Frontier for Natural Products." Chemistry \& Biology 5:R245-R249.

Harley, Isaac T.W., and Christopher L. Karp. 2012. "Obesity and the Gut Microbiome." Molecular Metabolism 1:21-31.

Hooks, Katarzyna B., and Maureen A. O'Malley. 2017. "Dysbiosis and its Discontents." mBio in press.

Hooper, Lora V., and Jeffrey I. Gordon. 2001. "Commensal Host-Bacterial Relationships in the Gut." Science 292:1115-1118.

Hooper, Lora V., Dan R. Littman, and Andrew J. Macpherson. 2012. "Interactions Between the Microbiota and the Immune System." Science 336:1268-1273.

Howick, Jeremy. 2011. "Exposing the Vanities - and a Qualified Defense - of Mechanistic Reasoning in Health Care Decision Making." Philosophy of Science 78:926-940.

Hsiao, Elaine Y., Sara W. McBride, Sophia Hsien, Gil Sharon, Embriette R. Hyde, et al. 2013. "Microbiota Modulate Behavioral and Physiological Abnormalities Associated with Neurodevelopmental Disorders." Cell 155:1451-1463. 
Huss, John. 2014. "Methodology and Ontology in Microbiome Research." Biological Theory 9:392-400.

Huttenhower, Curtis, Rob Knight, C. Titus Brown, J. Gregory Caporaso, Jose C. Clemente, et al. 2014. "Advancing the Microbiome Research Community." Cell 159:227-230.

Jeffery, lan B., Denise B. Lynch, and Paul W. O'Toole. 2016. "Composition and Temporal Stability of the Gut Microbiota in Older Persons." ISME Journal 10:170182.

Johnson, Elizabeth L., Stacey L. Heaver, William A. Walters, Ruth E. Ley. 2017. "Microbiome and Metabolic Disease: Revisiting the Bacterial Phylum Bacteroidetes." Journal of Molecular Medicine 95:1-8.

Justus, James. 2007. "Complexity, Diversity, and Stability." Pp. 321-350 in A Companion to the Philosophy of Biology. Edited by Sahotra Sahotra and Anya Plutynski. Oxford, UK: Blackwell.

Kassam, Zain, Christine H. Lee, Yuhong Yuan, and Richard H. Hunt. 2013. "Fecal Microbiota Transplantation for Clostridium difficile: Systematic Review and MetaAnalysis." American Journal of Gastroenterology 108:500-508.

Khoruts, Alexander, Johan Dicksved, Janet K. Jansson, and Michael J. Sadowsky. 2010. "Changes in the Composition of the Human Fecal Microbiome After Bacteriotherapy for Recurrent Clostridium difficile-Associated Diarrhea." Journal of Clinical Gastroenterology 44:354-360.

Knights, Dan, Kara G. Lassen, and Ramnik J. Xavier. 2013. "Advances in Inflammatory Bowel Disease Pathogenesis: Linking Host Genetics and the Microbiome." Gut 62:1505-1510.

La Caze, Adam. 2011. "The Role of Basic Science in Evidence-Based Medicine." Biology and Philosophy 26:81-98.

Lawley, Trevor D., Simon Clare, Alan W. Walker, Mark D. Stares, Thomas R. Connor, et al. 2012. "Targeted Restoration of the Intestinal Microbiota with a Simple, Defined Bacteriotherapy Resolves Relapsing Clostridium difficile Disease in Mice." PLOS Pathogens, 8(10):e1002995.

Lawley, Trevor D., and Alan W. Walker. 2012. "Intestinal Colonization Resistance." Immunology 138:1-11.

Layeghifard, Mehdi, David M. Hwang, and David S. Guttman. 2017. "Disentangling Interactions in the Microbiome: A Network Perspective." Trends in Microbiology 25:217-228.

Lepage, Patricia, Marion C. Leclerc, Marie Joossens, Stanislas Mondot, Hervé M. Blottière, et al. 2013. "A Metagenomic Insight into Our Gut's Microbiome." Gut 62:146-158. 
Levy, Maayan, Aleksandra A. Kolodziejczyk, Christoph A. Thaiss, and Eran Elinav. 2017. "Dysbiosis and the Immune System." Nature Reviews Immunolology 17:219232.

Lewis, James D., Eric Z. Chen, Robert N. Baldassano, Anthony R. Otley, Anne M. Griffiths, et al. 2015. "Inflammation, Antibiotics, and Diet as Environmental Stressors of the Gut Microbiome in Pediatric Crohn's Disease." Cell Host \& Microbe 18:489500 .

Ley, Ruth E., Peter J. Turnbaugh, Samuel Klein, and Jeffrey I. Gordon. 2006. "Human Gut Microbes Associated with Obesity." Nature 444:1022-1023.

Louca, Stilianos, Laura W. Parfrey, and Michael Doebeli. 2016. "Decoupling Function and Taxonomy in the Global Ocean Microbiome." Science 353:1272-1277.

Macpherson, Andrew J., Yasmin Köller, and Kathy D. McCoy. 2015. "The Bilateral Responsiveness Between Intestinal Microbes and IgA." Trends in Immunology 36:460-470.

Manor, Ohad, Roie Levy, and Elhanan Borenstein. 2014. "Mapping the Inner Workings of the Microbiome: Genome- and Metagenomics-Based Study of Metabolism and Metabolic Interactions in the Human Microbiome." Cell Metabolism 20:742-752.

Marchesi, Julian R. 2011. "Human Distal Gut Microbiome.” Environmental Microbiology 13:3088-3102.

Marino, Simeone, Nielson T. Baxter, Gary B. Huffnagle, Joseph F. Petrosino, and Patrick D. Schloss. 2014. "Mathematical Modelling of Primary Succession of Murine Intestinal Microbiota." Proceedings of the National Academy of Sciences U.S.A. 111:439-444.

McCann, Kevin S. 2000. "The Diversity-Stability Debate.” Nature 405:228-233.

Mendelsohn, J. Andrew. 2002. “'Like All That Lives': Biology, Medicine and Bacteria in the Age of Pasteur and Koch." History and Philosophy of the Life Sciences 24:336.

Momozawa, Yukihide, Valérie Deffontaine, Edouard Louis, and Juan F. Medrano. 2011. "Characterization of bacteria in biopsies of colon and stools by high throughput sequencing of the $\mathrm{V} 2$ region of bacterial 16S rRNA gene in human." PLOS One 6(2): e16952.

Moya, Andrés, and Manuel Ferrer. 2016. "Functional Redundancy-Induced Stability of Gut Microbiota Subjected to Disturbance." Trends in Microbiology 24:402-413.

Mullins, Thomas D., Theresa B. Britschgi, Robin L. Krest, and Stephen J. Giovannoni. 1995. "Genetic Comparisons Reveal the Same Unknown Bacterial 
Lineages in Atlantic and Pacific Bacterioplankton Communities." Limnology and Oceanography 40:148-158.

Noecker, Cecelia, Alexander Eng, Sujatha Srinivasan, Casey M. Thereiot, Vincent B. Young, et al. 2016. "Metabolic Model-Based Integration of Microbiome Taxonomic and Metabolomic Profiles Elucidates Mechanistic Links between Ecological and Metabolic Variation." mSystems 1(1):e00013-15.

Odenbaugh, Jay. 2007. "Seeing the Forest and the Trees: Realism about Communities and Ecosystems." Philosophy of Science 74: 628-641.

Olesen, Scott W., and Eric J. Alm. 2016. "Dysbiosis is not an Answer." Nature Microbiology 1:1-2.

Olsen, Gary J., David J. Lane, Stephen J. Giovannoni, and Norman R. Pace. 1986. "Microbial Ecology and Evolution: A Ribosomal RNA Approach." Annual Review of Microbiology 40:337-365.

O’Malley, Maureen A. 2014. Philosophy of Microbiology. Cambridge UK: Cambridge University Press.

O’Malley, Maureen A. 2008. "Exploratory Experimentation and Scientific Practice: Metagenomics and the Proteorhodopsin Case." History and Philosophy of the Life Sciences 29:337-358.

O’Malley, Maureen A., Ingo Brigandt, Alan C. Love, John W. Crawford, Jack A. Gilbert, et al. 2014. "Multilevel Research Strategies and Biological Systems." Philosophy of Science 81:811-828.

Parte, Aidan C. 2013. "LPSN - A List of Prokaryotic Names with Standing in Nomenclature." Nucleic Acids Research 42:D613-D616.

Petersen, Charisse, and June L. Round. 2014. "Defining Dysbiosis and its Influence on Host Immunity and Disease." Cellular Microbiology 16:1024-1033.

Prescott, Susan L. 2017. "History of Medicine: Origin of the Term Microbiome and Why it Matters." Human Microbiome Journal 4:24-25.

Rakoff-Nahoum, Seth, Kevin R. Foster, and Laurie E. Comstock. 2016. "The Evolution of Cooperation Within the Gut Microbiota." Nature 533:255-259.

Reid, Gregor, Jessica A. Younes, Henny C. Van der Mei, Gregory B. Gloor, Rob Knight, and Henk J. Busscher. 2011. "Microbiota Restoration: Natural and Supplemented Recovery of Human Microbial Communities." Nature Reviews Microbiology 9:27-38.

Relman, David A., and Stanley Falkow. 2001. "The Meaning and Impact of the Human Genome Sequence for Microbiology." Trends in Microbiology 9:206-208. 
Ridaura, Vanessa K., Jeremiah J. Faith, Federico E. Rey, Jiye Cheng, Alexis E. Duncan, et al. 2013. "Gut Microbiota from Twins Discordant for Obesity Modulate Metabolism in Mice." Science 341:1241214.

Ross, Lauren N., and James F. Woodward. 2016. "Koch's Postulates: An Interventionist Perspective." Studies in History and Philosophy of Biological and Biomedical Sciences 59:35-46.

Round, June L., and Sarkis K. Mazmanian. 2009. "The Gut Microbiota Shapes Intestinal Immune Responses During Health and Disease." Nature Reviews Immunology 9:313-323.

Schulberg, J., and P. De Cruz. (2016). "Characterisation and Therapeutic Manipulation of the Gut Microbiome in Inflammatory Bowel Disease." Internal Medicine Journal 46:266-273.

Schwabe, Robert F., and Christian Jobin. 2013. "The Microbiome and Cancer." Nature Reviews Cancer 13:800-812.

Schwiertz, Andreas, David Taras, Klaus Schäfer, Silvia Beijer, Nicolaas A. Bos, et al. 2010. "Microbiota and SCFA in Lean and Overweight Healthy Subjects." Obesity 18:190-195.

Seedorf, Henning, Nicholas W. Griffin, Vanessa K. Ridaura, Alejandro Reyes, Jiye Cheng, et al. 2014. "Bacteria from Diverse Habitats Colonize and Compete in the Mouse Gut.” Cell 159:253266.

Singleton, Rivers Jr., and David R. Singleton. 2016. "Remembering Our Forebears: Albert Jan Kluyver and the Unity of Life." Journal of the History of Biology 50:169218.

Skillings, Derek. 2016. "Holobionts and the Ecology of Organisms: Multi-Species Communities or Integrated Individuals." Biology and Philosophy 31:875-892.

Spor, Aymé, Omry Koren, and Ruth Ley. 2011. "Unravelling the Effects of the Environment and Host Genotype on the Gut Microbiome." Nature Reviews Microbiology 9:279-290.

Stahl, David A., David J. Lane, Gary J. Olsen, and Norman R. Pace, N. R. 1985. "Characterization of a Yellowstone Hot Spring Microbial Community by $5 S$ rRNA Sequences." Applied and Environmental Microbiology 49:1379-1384.

Stein, Jefferey L., Terence L. Marsh, Ke Y. Wu, Hiroaki Shizuya, and Edward F. DeLong. 1996. "Characterization of Uncultivated Prokaryotes: Isolation and Analysis of a 40-Kilobase-Pair Genome Fragment from a Planktonic Marine Archaeon." Journal of Bacteriology 178:591-599.

Surana, Neeraj K., and Dennis L. Kasper. 2017. "Moving Beyond Microbiome-Wide Associations to Causal Microbe Identification." Nature 552:244-247. 
Sze, Marc A., and Patrick D. Schloss. 2016. "Looking for a Signal in the Noise: Revisiting Obesity and the Microbiome." mBio 7 (4):e01018-16.

Tang, W. H. Wilson, and Stanley L. Hazen. 2014. "The Contributory Role of Gut Microbiota in Cardiovascular Disease." Journal of Clinical Investigation 124:42044211.

Taxis, Tasia M., Sara Wolff, Sarah J. Gregg, Nicholas O. Minton, Chiqian Zhang, et al. 2015. "The Players May Change but the Game Remains: Network Analyses of Ruminal Microbiomes Suggest Taxonomic Differences Mask Functional Similarity." Nucleic Acids Research 43:9600-9612.

Turnbaugh, Peter J., Bäckhed, F., Fulton, L., and Jeffrey I. Gordon. 2008. "DietInduced Obesity is Linked to Marked but Reversible Alterations in the Mouse Distal Gut Microbiome." Cell Host \& Microbe 3:213-223.

Turnbaugh, Peter J., Ruth E. Ley, Micah Hamady, Claire Fraser-Liggett, Rob Knight, and Jeffrey I. Gordon. 2007. "The Human Microbiome Project: Exploring the Microbial Part of Ourselves in a Changing World." Nature 449:804-810.

Turnbaugh, Peter J., Ruth E. Ley, Mahowald, M. A., Magrini, V., Mardis, E. R., and Jeffrey I. Gordon. 2006. "An Obesity-Associated Gut Microbiome with Increased Capacity for Energy Harvest." Nature 444:1027-1031.

Turnbaugh, Peter J., Ridaura, V. K., Faith, J. J., Rey, F. E., Knight, R., and Jeffrey I. Gordon. 2009. "The Effect of Diet on the Human Gut Microbiome: A Metagenomic Analysis in Humanized Gnotobiotic Mice." Science Translational Medicine 1(6):6ra14.

Tyson, Gene W., Jarrod Chapman, Philip Hugenholtz, Eric E. Allen, Rachna J. Ram, et al. 2004. "Community Structure and Metabolism Through Reconstruction of Microbial Genomes from the Environment." Nature 428:37-43.

Van Nood, Els, Anne Vrieze, Max Nieuwdorp, Susana Fuentes, Erwin G. Zoetendal, et al. 2013. "Duodenal Infusion of Donor Feces for Recurrent Clostridium difficile." New England Journal of Medicine 368:407-415.

Venter, J. Craig, Karin Remington, John F. Heidelberg, Aaron L. Halpern, Doug Rusch, et al. 2004. "Environmental Genome Shotgun Sequencing of the Sargasso Sea." Science, 304, 66-74.

Walters, William A., Zech Xu, and Rob Knight. 2014. "Meta-Analyses of Human Gut Microbes Associated with Obesity and IBD." FEBS Letters 588:4223-4233.

Wang, Zeneng, Elizabeth Klipfell, Brian J. Bennett, Robert Koeth, Bruce S. Levison, et al. 2011. "Gut Flora Metabolism of Phosphatidylcholine Promotes Cardiovascular Disease." Nature 472:57-63.

Whitham, Thomas G., Joseph K. Bailey, Jennifer A. Schweitzer, Stephen M. Shuster, Randy K. Bangert, et al. (2006) "A Framework for Community and Ecosystem Genetics: From Gene to Ecosystems." Nature Reviews Genetics" 7:510-523. 
Woese, Carl R., and George E. Fox. 1977. "Phylogenetic Structure of the Prokaryotic Kingdom: The Primary Kingdoms." Proceedings of the National Academy of Sciences U.S.A. 74:5088-5090.

Wu, Gary D., and James D. Lewis. 2013. "Analysis of the Human Gut Microbiome and Association with Disease." Clinical Gastroenterology and Hepatology 11:774777.

Yarza, Pablo, Pelin Yilmaz, Elmar Pruesse, Frank. O. Glöckner, Wolfgang Ludwig, et al. 2014. "Uniting the Classification of Cultured and Uncultured Bacteria and Archaea Using 16S rRNA Gene Sequences." Nature Reviews Microbiology 12:635-645.

Zhang, Husen, John K. DiBaise, Andrea Zuccolo, Dave Kudrna, Michele Braidotti, M., et al. 2009. "Human Gut Microbiota in Obesity and After Gastric Bypass."

Proceedings of the National Academy of Sciences U.S.A. 106:2365-2370.

Zhao, Liping. 2013. "The Gut Microbiota and Obesity: From Correlation to Causality." Nature Reviews Microbiology 11:639-647.

Zhu, Weifei, Jill C. Gregory, Elin Org, Jennifer A. Buffa, Nilaksh Gupta, et al. 2016. "Gut Microbial Metabolite TMAO Enhances Platelet Hyperreactivity and Thrombosis Risk." Cell 165:111-124.

Zoetendal, Erwin G., Chad T. Collier, Satoshi Koike, Roderick I. Mackie, and H. Rex Gaskins. 2004. "Molecular Ecological Analysis of the Gastrointestinal Microbiota: A Review." Journal of Nutrition 134:465-472. 